\title{
Postpartum Complication
}

National Cancer Institute

\section{Source}

National Cancer Institute. Postpartum Complication. NCI Thesaurus. Code C116323.

Any adverse event that arises in the six week period immediately following the delivery of a fetus. 